\title{
LA PROTECCIÓN DEL USUARIO DEL SERVICIO PÚBLICO DE ENERGÍA ELÉCTRICA EN LA RECIENTE JURISPRUDENCIA DEL SUPERIOR TRIBUNAL DE JUSTICIA DE LA PROVINCIA DE CORRIENTES ${ }^{1}$
}

Miguel Andrés Goldfarb ${ }^{2}$

\section{Introducción}

En el presente trabajo expondré acerca del avance jurisprudencial que en materia de protección de usuarios de servicios públicos ante la administración se viene dando en la provincia de Corrientes a partir de la Reforma Constitucional Provincial del año 2007. A tales fines tomo como puntos de análisis dos fallos relevantes del Superior Tribunal de la citada Provincia, de cuyos fundamentos y resoluciones se desprenden una serie elementos harto relevantes en materia de tutela de las personas cuando ocupamos el rol de usuarios de un servicio público esencial.

Con respecto a la trascendencia del tema es necesario tener presente que el campo de los servicios públicos constituye una sensible área de gobierno en donde se concretiza la acción pública. Es aquí en donde la relación ciudadanoEstado se estrecha, se plasma y se edifica con fuerza y nitidez.

Además, la realidad del siglo XXI nos muestra que los servicios públicos de hoy constituyen mecanismos de igualación social directamente vinculados con calidad de vida y derechos humanos. De tal forma, el acceso igualitario al agua, energía y transporte debe buscar mecanismos administrativos que se enmarquen en el espíritu que las constituciones le han impregnado a esta temática. (artículos 42 y 48 de las Constituciones de Argentina y Corrientes respectivamente).

${ }^{1}$ Trabajo oportunamente aceptado para ser incluido como documento a publicarse en el congreso internacional del CLAD, Montevideo, 2013.

${ }^{2}$ Docente categorizado investigador de las asignaturas de Derecho Financiero y Tributario y Derecho Administrativo. Especialista en Docencia Universitaria (UNNE), Asesoría de Empresas (UBA), Derecho Administrativo (UNNE) y Master en Derecho Tributario (Universidad de Barcelona). Doctorando en Derecho Público, UNNE. 
En virtud de lo expuesto, la tutela de los derechos de los usuarios, el control judicial del ejercicio de poder público y las prerrogativas que ejercen la administración toman hoy una importancia notable que se manifiesta en la realidad cotidiana y práctica de millones de ciudadanos en un contexto de desigualdad social y problemáticas irresueltas de diversa índole.

Previamente a analizar los dos precedentes objeto de este documento, abordo conceptualmente el plano constitucional de la materia, en virtud del cual, desde 1994 en la Nación y 2007 en Corrientes el usuario tiene una serie de derechos fundamentales en el marco de las relaciones de consumo. Este tópico es relevante toda vez que en estas regulaciones constitucionales se sostienen luego los fallos a comentar.

En el primer caso judicial que trato se resolvió la inconstitucionalidad de un cuadro tarifario que dispuso un aumento retroactivo en la tarifa eléctrica y que fue aprobado por la administración sin la realización de la audiencia pública que la constitución ordena. El nuevo modelo tarifario proponía además un sistema proporcional de escalonamiento de tasas que no se condice con el principio de igualdad en la materia. Me permito allí hacer un breve análisis sobre las facultades discrecionales y el control judicial sobre el ejercicio de ellas, tema largamente debatido en la doctrina administrativista.

En la parte medular de la sentencia que me ocupa, en primer término, se puso sobre relieve el deber constitucional de participación de los usuarios a través de las audiencias públicas, aun cuando el artículo que la prescribe no se encontraba reglamentado. Hay además una interesante fundamentación del rol de los jueces en el control de constitucionalidad y en la revisión de los actos de la administración.

En el segundo precedente, también emanado del Superior Tribunal de Justicia correntino, se ordenó una franquicia del $100 \%$ en la tarifa eléctrica a favor de una persona de escasos recursos que tiene a su cargo un hijo menor de edad con discapacidad. El fallo de primera instancia fue luego confirmado por el Máximo Tribunal Provincial.

Se planteó allí la inconstitucionalidad del Reglamento de Suministro que establece el corte del servicio en el caso de mora en el pago de la factura. El derecho a la salud viene a ocupar aquí un papel primordial en el análisis de los jueces.

Los magistrados deben ponderar y valorar derechos que surgen del texto de la Carta Magna y de los Tratados que poseen también jerarquía constitucional. 
Finalmente expondré las conclusiones acerca del perfil que viene adoptando la jurisprudencia analizada, sin perder de vista que se tratan de casos reales en donde la dignidad humana y las condiciones de vida apropiadas le dan vida a las normas jurídicas plasmadas en los boletines oficiales.

\section{El usuario en la Constitución}

La Reforma Constitucional Argentina del año 1994 receptó criterios emanados de las ciencias jurídicas -y del derecho comparado- (como la categorización constitucional de derechos en relaciones de consumo), y por la otra, su objetivo fue -y es- generar nuevos escenarios sociales y humanos. Lo ha logrado hasta el momento. Es decir, nuestro artículo 42 es causa y efecto a su vez de una "nueva realidad jurídica”. Sus efectos aún se encuentran en proceso y es tarea nuestra y de la jurisprudencia precisarlos.

Se conformó un verdadero estatuto constitucional a partir de la consagración del citado artículo 42 que "positivizó” con rango supremo los derechos de los usuarios y consumidores, creando, tal como lo veremos, un microcosmos jurídico en la materia.

María Angélica Gelly (2006:462) explica que el citado artículo 42 tiene tres objetivos definidos: protección del consumidor, otorgar garantías a los competidores (libre concurrencia) y procurar la transparencia del mercado; este último a favor de oferentes y demandantes.

Partiendo de lo expresado en la primera parte de este capítulo es dable afirmar que el texto constitucional importó la positivización de una serie de principios jurídicos tendientes a proteger y defender al usuario en un contexto de economía "social de mercado". Asimismo, el dato relevante es que este categorización constitucional irradia sobre el todo el subsistema de reglas y principios que regulan a la persona en su faceta de consumidor o usuario. Enfocaré la cuestión desde el punto de vista de este último.

El texto del artículo brinda un trato indistinto a consumidores y usuarios y este aspecto es central, pues su equiparación gira sobre la idea que siempre son personas, sin perjuicio del tipo de relación que entablen con proveedores de bienes y servicios, o la naturaleza de estos.

El citado artículo también fija una serie de principios en materia de regulación de los servicios públicos. 
Al margen de la titularidad estatal o no de los servicios públicos, lo cierto es que en la praxis jurídica el Estado tiene ahora un mandato expreso, cuyo alcance debe ser delimitado a la luz de una correcta, integral y armónica interpretación del texto constitucional. Este hilo conductor nos ha de llevar hasta la figura central del usuario.

El Capítulo Segundo de nuestra Constitución recogió los denominados derechos de tercera generación, entre los que se ubicaron aquellos vinculados con la materia ambiental, las relaciones de consumo, la participación ciudadana y la expresa regulación del amparo como mecanismo de garantía contemplado en el conocido artículo 43. En el artículo 42 se le dio trato al usuario y a los servicios públicos. ${ }^{3}$

El constituyente creó así un microsistema comprensivo de materias nuevas que tomaron trascendencia social en las últimas décadas. En ese ámbito, encontramos hoy la regulación constitucional que paso a analizar. La reforma constitucional de Corrientes incorporó en su artículo 48 un mandato análogo al hasta aquí analizado. Queda así planteado el escenario.

\section{La revisión judicial de las tarifas de servicios públicos}

En el primer caso que aquí se analizará se pone bajo el análisis un fallo emanado del Superior Tribunal de Justicia de la Provincia de Corrientes ${ }^{4}$ en virtud del cual, confirmando la sentencia dictada en primera instancia, se declaró la inconstitucionalidad de un aumento tarifario dispuesto en materia eléctrica

${ }^{3}$ Cabe citar el artículo 42: los consumidores y usuarios de bienes y servicios tienen derecho, en la relación de consumo, a la protección de su salud, seguridad e intereses económicos; a una información adecuada y veraz; a la libertad de elección, y a condiciones de trato equitativo y digno. Las autoridades proveerán a la protección de esos derechos, a la educación para el consumo, a la defensa de la competencia contra toda forma de distorsión de los mercados, al control de los monopolios naturales y legales, al de la calidad y eficiencia de los servicios públicos, y a la constitución de asociaciones de consumidores y de usuarios. La legislación establecerá procedimientos eficaces para la prevención y solución de conflictos, y los marcos regulatorios de los servicios públicos de competencia nacional, previendo la necesaria participación de las asociaciones de consumidores y usuarios y de las provincias interesadas, en los organismos de control.

${ }^{4}$ Asociación de Usuarios y Consumidores de la Provincia de Corrientes c/Dirección Provincial de Energía Eléctrica s/amparo. expte. 32226/9 www.juscorrientes.gov.ar 
sin la previa realización del mecanismo participativo de audiencia pública previsto en la -en su momento recientemente reformada- Constitución Provincial.

Mediante el Decreto Provincial Nº 2668/08 el Poder Ejecutivo Corrientes, ratificando la Resolución 1049/08 de la Dirección Provincial de Energía Eléctrica (DPEC) estableció un nuevo cuadro tarifario para el servicio en cuestión. ${ }^{5}$

El nuevo sistema tarifario se caracterizó por instrumentar un mecanismo progresivo de tasas de "pequeñas demandas residenciales" con un cargo fija $(\$ 13,20)$ y luego escalonadamente tarifas en sentido ascendente conforme el nivel de consumo. Por ejemplo, de 0 a $500 \mathrm{KW} / \mathrm{h}$ bimestrales 0,164 centavos, desde 501 a $1000 \mathrm{KW} / \mathrm{h} 0,19$ y así sucesivamente.

La cuestión es que a partir de $1000 \mathrm{KW} / \mathrm{h}$ la tarifa se fijó de forma proporcional y directa para todo el consumo en $0,26 \mathrm{KW} / \mathrm{h}$, borrando así todo el sentido de progresividad tarifaria. Desde $1400 \mathrm{KW} / \mathrm{h}$ el costo establecido fue de 0,3102 centavos. El mecanismo se reitera para las demás categorías tales como demandas por servicios generales.

El cuadro tarifario mantuvo la tarifa social oportunamente creada en el año 2002 a través de la resolución 205 de aquel año, con a un cargo fijo de ocho pesos con tres centavos $(\$ 8,03) \mathrm{y}$, para aquellos usuarios de hasta $300 \mathrm{KW} / \mathrm{h}$ 0,05 centavos y 0,06 para aquellos que consumen hasta $500 \mathrm{KW} / \mathrm{h}$.

Cassagne (2006:433) señala que el principio de proporcionalidad de las tarifas se expresa en que estas deben ser justas y razonables. Los justo se relacionaría con aspectos jurídicos, tales como los modos de aplicar la tarifa (generalidad e igualdad) y lo razonable con el quantum. Claro está, ambos son conceptos indeterminados, pero que nos indican que las tarifas deben surgir de una ecuación equilibrada con el costo del servicio y una utilidad tasada y proporcional.

La primera cuestión que se suscita se relaciona con la posibilidad de que los jueces entren a valorar la cuantía de la tarifa. Se juega aquí el ámbito de reserva y discreción administrativa y el principio republicano de control judicial.

Me permito aquí hacer algunas apreciaciones sobre estos tópicos para retomar luego la estructura argumental del fallo que comento. En tal sentido, vale recor-

${ }^{5}$ Es dable recordar que las tarifas constituyen un precio público, al decir de Gaspar Ariño Ortiz (2005:315) un justiprecio por la prestación de un servicio público. Compartimos la posición de Ariño Ortiz en tanto sostiene que la aprobación de una tarifa constituye un acto jurídico que goza de carácter normativo y que emana del gobierno o una agencia o ente regulador independiente y que, por lo tanto, debe someterse al derecho. 
dar la discrecionalidad que tiene la administración para fijar tarifas se ve sensiblemente acotada, permitiendo en algunos casos solo un relativo margen de valoración para el órgano entre las opciones posibles. Volveremos luego sobre este punto.

En esta materia, a la luz del mandato Constitucional de la Provincia (artículo 48) también existen otros mecanismos tendientes a asegurar la participación y los derechos del universo de usuarios afectados, tales como las audiencias públicas y los entes reguladores.

Se asocia el concepto de "lo discrecional" con cierto margen libertad que el órgano posee legítimamente para resolver o actuar ante un determinado caso.

Cassagne (2006:223) reconoce al menos tres modalidades de discrecionalidad administrativa, a saber: a) casos en los cuales el margen de arbitrio no está limitado por conceptos jurídicos indeterminados (discrecionalidad pura o típica), b) aquella en la cual se apelan a estos conceptos jurídicos como fórmulas limitativas de la discreción, ubicando aquí el caso de las tarifas de servicios públicos concesionados en tanto normalmente se prescribe que estas deben ser "justas y razonables"; y c) discrecionalidad ceñida a presupuestos dados por la norma objetiva. Nuestro caso se ajustaría al segundo supuesto ("tarifas justas y razonables").

El concepto de discrecionalidad en modo alguno puede asociarse a inmunidad zona de reserva absoluta, pues en el contexto del estado Constitucional de Derecho no existe margen para que la actividad estatal quede sujeta al arbitrio de un funcionario o de un órgano ni exenta de control judicial. Discrecionalidad no es arbitrariedad.

Al respecto, hago propia la posición de la Profesora Mirta Sotelo de Andreau cuando claramente postula que

la discrecionalidad no implica de ninguna manera la libertad amplia frente a la norma, es en realidad la posibilidad de valoración de las circunstancias particulares en cada caso concreto, otorgándose un margen de posibilidades en aplicación a las autoridades de la Administración en el marco de sus políticas de progreso.

Delimitado el concepto de discrecionalidad, es dable tener presente que la doctrina contemporánea y a todas luces correcta, concuerda en que tales facultades no constituyen poderes al margen de la jurisdicción ni del control público puesto que no constituyen atribuciones que el sistema normativo le reconoce a un órgano administrativo exentas de toda posibilidad de revisión judicial. 
Todos los actos emanados del poder administrador, son pues, susceptibles de control y revisión judicial al amparo del mandato de los artículos 116 y 117 de la Constitución Nacional. ${ }^{6}$

Ahora bien, fijado el principio rector en el marco del estado de derecho, la cuestión práctica será delimitar en cada caso concreto las posibilidades fácticas reales del control judicial a efectos de evitar el gobierno de los jueces, es decir, un desplazamiento no querido por el sistema constitucional y un exceso de tales atribuciones. En la práctica, ante planteos judiciales que pretenden la declaración de "excesiva o arbitraria” de una determinada tarifa, las dificultades del Juez para sentenciar y valorar adecuada y fundadamente tales apreciaciones son por demás excesivas.

La existencia de complejas fórmulas polinómicas y la falta de información disponible constituyen en no pocas ocasiones verdaderos obstáculos para el sentenciante, y así, muchos fallos terminan acudiendo a cuestiones o vicios procedimentales para invalidar una tarifa. ${ }^{7}$

Por su parte, la doctrina ha evolucionado favorablemente, partiendo de la negativa y aceptando en la actualidad la revisión de los tribunales. La ruptura de la proporcionalidad de la tarifa autoriza al usuario afectado a impugnar judicialmente el acto de alcance general de fijación de la tarifa, o bien el acto particular de su aplicación. Como se verá infra, este ha sido el caso que tuvo que resolver la corte correntina.

En los párrafos subsiguientes veremos de qué modo el Superior Tribunal de Justicia de Corrientes ha sentenciado una compleja cuestión, ejerciendo así la difícil tarea de revisar actos dictados en ejercicio de facultades discrecionales en materia de tarifas de servicios públicos.

${ }^{6}$ Sostiene Cassagne (2006:227) que en nuestro sistema jurídico rige el principio de plena judiciabilidad de las causas en que son parte las diferentes administraciones (arts. 116 117, CN) particularmente cuando se trata del juzgamiento de controversias cuyo objeto central es la revisión de actos y reglamentos administrativos. De otro modo, no existiría la tutela o protección judicial efectiva.

${ }^{7}$ Al respecto señala Urrutigoity (1994:91) que "en última instancia, el resultado del control no podrá consistir en sustituir a la administración en la fijación de una tarifa suficiente. Como ya dije, vendrá dado por el otorgamiento de una compensación que garantice al gestor la ecuación económica financiera, la relación inversión rentabilidad, la intangibilidad de la remuneración que el contrato le garantiza". 


\section{El primer caso judicial}

Ante el incremento de la tarifa del servicio eléctrico, la Asociación de Usuarios y Consumidores de la Provincia de Corrientes solicitó la declaración de la invalidez constitucional de la Resolución No 1049/08 y el Decreto N ${ }^{\circ}$ 2668/08 fundando su posición en la excesiva y desproporcionada facturación del consumo de energía eléctrica, que según informan los tornó manifiestamente ilegales e irrazonables, además de haber violado el artículo 48 de la Constitución Provincial por la falta de participación de los interesados, consumidores y usuarios, en una audiencia pública convocada al efecto, y por haber desconocido también las disposiciones contenidas en los artículos 28 y 42 de la Constitución Nacional. ${ }^{8}$

El Juez de Primera Instancia hizo lugar a la acción de amparo promovida y estableció la inconstitucionalidad del aumento tarifario basándose en los siguientes puntos: a) su retroactividad (al primero de octubre del 2008) y b) consideró no respetó el derecho de información que le asiste a los usuarios del servicio.

En lo que atañe a la revisión judicial de tarifas desde el punto de vista económico consideró que "no correspondía analizar si el precio era excesivo o no" pero sí al aumento de grosero y perjudicial para los usuarios, toda vez que el aumento aplicable a los consumos superiores a 1000 kilovatios por bimestre era extensivo a toda la escala de modo directo. Sentenció en congruencia con lo señalado que el incremento tarifario era desproporcionado, ilegítimo y arbitrario.

Analizando los aspectos de fondo, uno de los motivos de la apelación del estado, se sustentó en que la sentencia era nula por ser "extra petita" dado que el aquo habría introducido la cuestión de la retroactividad de la nueva tarifa como elemento inconstitucional que la invalidaba.

Llegada la causa al Superior Tribunal de Justicia el primer elemento de juicio en el recurso fue que la cuestión de la "retroactividad" de las nuevas tarifas no había sido reclamada por la actora, razón por la cual, la sentencia era "extra petita".

Con interesantes párrafos, el Máximo órgano judicial justificó su potestad revisora y argumentó plenamente el deber jurisdiccional de velar por la

${ }^{8}$ Asociación de Usuarios y Consumidores de la Provincia de Corrientes c/ Dirección Pcial. de Energía de Corrientes y Estado de la pcia. de Ctes. s/ amparo expte. nº 32226/9 
constitucionalidad del orden jurídico, aun cuando no lo hubiera pedido la parte. Se desestimó así el primer argumento de la demandada en su recurso. ${ }^{9}$

Consideró además el Tribunal que el control de justicia y razonabilidad de las tarifas debe ser amplio y no limitado. Luego ponderó que

En ese particular contexto, y más allá del juzgamiento de la razonabilidad o irrazonabilidad del nuevo cuadro tarifario implementado por la Dirección Provincial de Energía por resolución $N^{o} 1049 / 08$ aprobado por decreto $N^{o} 2668 / 08$, que de acuerdo a los fundamentos expresados por los accionados tuvo como antecedente inmediato la resolución $N^{\circ}$ 1169/08 de la Secretaría General de la Nación, lo cierto y concreto es que la Dirección Provincial de Energía única prestataria de este servicio público en el ámbito provincial, no ha seguido con el procedimiento constitucional establecido al efecto, tornando a estos actos del poder administrador contrarios a la Constitución de la Provincia y a preceptos de la Constitución Nacional.

Ahora bien, el eje de la sentencia se presenta en la impugnación constitucional del cuadro tarifario en tanto y en cuanto este se aprobó obviando la convocatoria y realización de la audiencia pública exigida por la Norma Fundamental desde su reforma en 2007.

El hecho de a la fecha no se encontrara reglamentado el artículo 48 de la Constitución - derechos de los usuarios y consumidores- no es óbice para el cumplimiento de tal mandato, toda vez que estos son operativos y no requieren tal reglamentación. ${ }^{10}$

${ }^{9}$ Dijo al respecto el tribunal: "en ese contexto considero que en la ineluctable misión de los magistrados de ejercer eficazmente el control de constitucionalidad de las normas, no pueden quedar ceñidos a las argumentaciones de las partes, más aún, no resulta necesario siquiera supeditar la invalidación de una norma evidentemente contraria al orden constitucional a la circunstancia que haya sido peticionada, pues ello no se condice con la función de la magistratura cuya misión esencial e indelegable consiste en aplicar el derecho (iura novit curia), no de cualquier forma, sino constitucionalmente" www.juscorrientes.gov.ar

${ }^{10}$ En este punto el fallo sostuvo que "la audiencia pública comporta un mecanismo de debate sobre diversos aspectos de la prestación de un servicio público y permite la democratización de las decisiones, máxime cuando, como en el caso, se trata de un significativo aumento del cuadro tarifario por la prestación del servicio de energía eléctrica, quedando de tal modo a salvo el derecho de defensa de los usuarios y consumidores, además de transparentar los 
No habiéndose llevado a cabo los procedimientos constitucionales para la readecuación de las tarifas, la sentencia confirma el fallo de primera instancia y declaró inconstitucional el nuevo cuadro tarifario.

Plasmado el principio de revisión judicial amplio en la sentencia comentada, encontramos que el análisis de los jueces se centra en los aspectos reglados por la norma y no en la sustancia discrecional.

Precisamente, en primera instancia, se hace hincapié en la retroactividad de la norma como vicio constitucional, se afirma la irrazonabilidad de la tarifa pero no se la desmenuza en su faceta financiera. Posteriormente, en la instancia de apelación se confirma la sentencia tomando en fuerte consideración la violación de los procedimientos constitucionales. Si bien se sostiene que el poder de revisión es amplio, nuevamente este no recae estrictamente sobre la razonabilidad de la cuantía del precio o tasa, sino sobre cuestiones regladas, en este caso, el deber procesal de realizar audiencia pública. Comparto los fundamentos del Tribunal pero hago notar que no se adentra en la ecuación financiera de la tarifa para juzgar con precisión su ilegitimidad.

\section{El segundo caso: tarifas y derecho a la salud en la prestación de los servicios públicos esenciales}

En el más que reciente precedente "Meza"11 emanado del Máximo Tribunal de la Provincia de Corrientes, el actor promovió una acción de amparo

procedimientos administrativos. la circunstancia de que no se encuentre reglamentado en el ámbito provincial en nada impide su plena operatividad, pues las garantías constitucionales existen y protegen a los individuos por el solo hecho de estar en la constitución e independientemente de sus leyes reglamentarias, cuyas limitaciones no pueden constituir obstáculo para la vigencia efectiva de dichas garantías. sobre la base de estas consideraciones arribo a la conclusión que la dirección provincial de energía al haber obviado el procedimiento de las audiencias públicas previsto expresamente en el art. 48 de la constitución provincial y de modo implícito en art. 42 de la constitución nacional, para la implementación del nuevo cuadro tarifario, además vulnerar los preceptos constitucionales sindicados, desconoció el derecho de defensa de los usuarios y consumidores del servicio, quienes se vieron privados de la posibilidad de conocer en profundidad y transparencia las razones que llevaron a la entidad accionada a disponer el aumento, convirtiendo a las resoluciones administrativas impugnadas desprovistas de toda validez constitucional."

${ }^{11}$ Meza Marta c/Dirección Provincial de Energía Eléctrica de la Pcia. de Corrientes y Estado de la Provincia de Corrientes s/amparo expte 40180. www.juscorrientes.gov.ar 
contra la Dirección de Energía Eléctrica a efectos de que se declare la inconstitucionalidad de una serie de artículos del Reglamento General de Suministro Eléctrico -aprobado oportunamente mediante Resolución $N^{\circ}$ 694/03 del mismo ente- en virtud de los cuales, se establecía respectivamente el deber del usuario de pagar las facturas y en el supuesto de incumplimiento, el corte de suministro sin necesidad de interpelación alguna.

El objeto de la acción era obtener una franquicia en el pago del servicio eléctrico con base en el derecho constitucional de la salud y la imposibilidad económica de afrontar el pago de las tarifas. Ni siquiera, la denominada "tarifa social”, oportunamente establecida por la administración mediante el Decreto, del suministro del servicio público de energía eléctrica implicaba una severa restricción sanitaria con consecuencias negativas para la salud del menor discapacitado. Se plantea allí el tema deciden dum: la concesión o no de una franquicia tarifaria para un caso excepcional en donde se pudiera afectar el derecho a la salud y, eventualmente, los alcances de esta.

En primera instancia, el Juzgado Civil y Comercial $N^{\circ} 7$ de la Ciudad de Corrientes hizo lugar a la acción deducida, declaró la inconstitucionalidad de los artículos antes señalados del Reglamento de Suministro Eléctrico y concedió una franquicia del $100 \%$ en el pago de la tarifa de energía liquidada por la empresa, siempre y cuando esta se correspondiera con un uso "básico y elemental” conforme al tabla que elabora la propia Dirección Provincial de Energía Eléctrica.

Entre sus fundamentos, este primer fallo se sostuvo en "cuestiones humanitarias” y el beneficio de competencia que surge de los artículo 799 y 800 del Código Civil. Asimismo, invitó a la accionada a contemplar casos como aquel para eximirlos del pago.

Contra esta sentencia, la accionada interpuso un recurso que dio lugar al fallo del Superior Tribunal de Justicia que infra comento.

Finalmente, la Corte Correntina estableció la inconstitucionalidad de los artículos mencionados del Reglamento de Suministro de Energía, por cuanto la exigencia en el cobro de las tarifas como presupuesto para la provisión de energía para personas en estado de necesidad y vulnerabilidad sanitaria afecta -primordialmente- un derecho constitucional fundamental, cual es, el derecho a la salud; directa y estrechamente vinculado con el derecho a la vida.

En su razonamiento, el citado Tribunal se sostuvo en tres pilares argumentales para confirmar el contenido del fallo dictado por el aquo: 
a) El mandato del artículo 42 de la Constitución Nacional Argentina, por cuanto prescribe la tutela de la salud, seguridad e intereses económicos de usuarios y consumidores en el marco de la relación de consumo, así como la protección en su participación en el mercado a través del trato digno, educación etc. y la regulación de los monopolios naturales y legales. Tal como lo indiqué ut supra, la Constitución Provincial replicó este artículo a partir de su Reforma en el año 2007, por lo que se vino así a "reforzar” esta cláusula constitucional incorporada en el plano nacional en 1994.

b) En el caso, el Tribunal contempló que

la salud y seguridad son propias del poder de policía del Estado, que según el artículo citado, debe tratar de resguardar la igualdad en el negocio jurídico entre los consumidores y los intereses de los proveedores del mercado de bienes y servicios.

c) El rango constitucional del derecho a la salud: De todos modos el aspecto más relevante del fallo ni su parte medular no constituyen lo sustentado por el artículo 42 de la C.N dado que la razón argumental central por la cual se confirmó la inconstitucionalidad del reglamento cuestionado radica en una colisión de derechos que se confrontan y que puede subsumirse del siguiente modo: derecho a la percepción de la tarifa vs. derecho a la salud. Es allí donde los jueces nutren su razonamiento y, en visión que comparto, ponderan las consideraciones especiales del caso, el derecho a la salud y las prescripciones administrativas emanadas del reglamento en cuestión.

Claro está que para así valorar, resulta indispensable tener por acreditados los extremos fácticos que dieron lugar a la vía del amparo, como lo son las condiciones de extrema pobreza y además, el hecho de que la actora tenía a su cargo un hijo menor discapacitado.

En el contexto referido, al Tribunal postuló que en el plano provincial, el derecho establecido por la Constitución a favor de los consumidores de energía eléctrica se encuentra limitado -en este caso de modo inconstitucional- por el Reglamento General de Suministro Eléctrico, el cual, tal como lo señalé ut supra, le concede a la prestataria del servicio público el poder de suspenderlo sin necesidad de previo aviso ni interpelación judicial o extrajudicial cuando medie mora en el pago de facturas, toda vez que la provisión de energía eléctrica es un servicio público de carácter oneroso, que no se presume gratuito y el corte del suministro ante el incumplimiento de pago del usuario es su resultado. 
Otro punto relevante se relaciona con la existencia de la tarifa social. Este dato no puede ser soslayado en el análisis del caso, por lo que, de forma también acertada, el fallo se refirió a este punto.

El interrogante que cabe efectuarse es el siguiente: A los fines de salvaguardar los derechos de la actora ¿no hubiera sido suficiente desde el punto de vista humanitario aplicar a este caso la llamada "tarifa social"?

El Tribunal consideró que el hecho de que existiera un régimen diferenciado en virtud del cual, mediante el Decreto 1289/02 se estableció una tarifa social de menor cuantía y una serie de facilidades en el pago la tarifa no salva la inconstitucionalidad de las normas administrativas impugnadas dado que en virtud de las excepcionales circunstancias del caso

está comprometido el derecho a la salud del hijo de la actora, derecho que desde el punto de vista normativo, está reconocido en los tratados internacionales con rango constitucional (art. 75 inc. 22, Constitución Nacional), entre ellos, el art. 12 inc.

(...) c) del Pacto Internacional de Derechos Económicos, Sociales y Culturales; inc. $1^{\circ}$ arts. $4^{\circ}$ y $5^{\circ}$ de la Convención sobre Derechos Humanos -Pacto de San José de Costa Rica- e inc. $1^{\circ}$ del art. $6^{o}$ del Pacto Internacional de Derechos Civiles y Políticos; y que la Corte desde su sinicios entendió que el Estado Nacional está obligado a "proteger la salud pública» (Fallos: 31:273) pues el derecho a la salud está comprendido dentro del derecho a la vida que es «el primer derecho natural de la persona humana preexistente a toda legislación positiva que, obviamente, resulta reconocido y garantizado por la Constitución Nacional.» (Fallos: 302:1284; 310:112 -La Ley, 1981-A 401; 1987B, 311)

En este sentido, vale recordar -en consonancia con el fallo- que el Preámbulo de la Constitución Nacional "ya se encuentran expresiones referidas al bienestar general, objetivo preeminente en el que, por cierto, ha de computarse con prioridad indiscutible, la preservación de la salud" (Fallos: 278:313, consid. 15).

d) Es política de Estado: En su tercera línea argumentativa, la sentencia nos remite a la Ley 24901 en virtud de la cual se instituyó un sistema de protección integral para las personas con discapacidad. Se estableció allí régimen especial de derechos de las personas con diferentes tipos de discapacidades y 
la consiguiente obligación a cargo de los órganos del Estado, tratando de otorgar a quienes se encontraren en esas condiciones, prerrogativas y estímulos a fin de intentar contrarrestar el menoscabo que la discapacidad les provoca (CSJN, Fallos: 313:579).

Por consiguiente, el rol del Estado en cualquiera sus facetas, impone un irrestricto deber de preservar el derecho a la salud -sinónimo de vida- por sobre toda otra cuestión. El preámbulo nacional, los artículos específicos vinculados con los derechos de usuarios y consumidores de bienes y servicios y el plexo de Tratados Internacionales que gozan hoy de jerarquía constitucional (art.75 inciso 22 de la Constitución Argentina) así lo exigen.

\section{Conclusiones}

Cabe concluir que de los fallos expuestos se denota una clara tendencia jurisprudencial en materia de protección de usuarios de servicios públicos locales en la provincia de Corrientes -República Argentina-.

En tal sentido, los derechos a la tutela de la salud, intereses económicos, educación, trato digno y equitativo etc. son plenamente operativos.

La exigencia judicial de realización de audiencia pública con participación de usuarios demuestra la intención de garantizar para estos el papel relevante que el constituyente le pretendido reconocer a los fines de la autoprotección de sus intereses.

Ahora bien, de las situaciones descriptas es dable afirmar también otros dos conceptos muy importantes. En primer lugar una noción amplia de tutela judicial que surge de la posibilidad de revisión jurisdiccional de las tarifas que fija la administración y la posibilidad de fallar "extra petita" cuando de derechos y garantías constitucionales se trata. Comparto este saludable criterio en un marco de sentido republicano de gobierno.

En segundo término la ponderación de derechos constitucionales que emana del segundo fallo analizado -en su caso la protección a ultranza del derecho a la salud y a la vida sobre el derecho al cobro de un servicio público- focaliza la atención en el principio de centralidad de la vida humana.

Así, proteger al usuario de un servicio esencial, garantizándolo cuando circunstancias extremas le impiden abonar el coste de aquel, implica el reconocimiento tácito de un supra principio que irradia sobre todo el sistema jurídico y que se vincula con la idea de protección integral de la persona humana. 
Al fin y al cabo, el derecho constituye un mecanismo social, un objeto cultural, cuya única finalidad es contribuir a la paz social a partir de "darle a cada uno lo suyo" equitativamente.

En los precedentes analizados considero que se plasma nítidamente una tendencia tuitiva y saludable de tales derechos, por lo que es dable afirmar que el mandato del constituyente se viene afirmando sobre la realidad.

Tal como lo señalé ab initio, el campo de los servicios públicos constituye área fértil para vislumbrar la forma y los alcances del ejercicio del poder público, el control judicial y el grado de protección que tenemos los ciudadanos en un contexto social cada vez más complejo y dinámico de manera tal que estas actividades públicas reflejan en el día a día el funcionamiento del Estado, cual gestor del bien común y garante de la plena realización individual.

\section{Bibliografía}

Ariño Ortiz, Gaspar (2005), Servicios Públicos. Regulación y Renegociación. Lexis Nexis Abeledo Perrot, Buenos Aires.

Cassagne, Juan Carlos (2006), Derecho Administrativo. Tomo II. Lexis Nexis, Buenos Aires.

Gelly, María Angélica (2006), Constitución de la Nación Argentina. Comentada y Actualizada. La Ley, Buenos Aires.

Urrutigoity, Javier (1994), Retribución de los Servicios Públicos en Los Servicios Públicos. Depalma, Buenos Aires.

Causa judicial Asociación de Usuarios y Consumidores de la Provincia de Corrientes c/ Dirección Pcial. de Energía de Corrientes y Estado de la Pcia. de Ctes. s/ Amparo Expte. No 32226/9 consultado el 25/04/13.

Causa judicial Meza Marta c/Dirección Provincial de Energía Eléctrica de la Pcia. de Corrientes y Estado de la Provincia de Corrientes s/Amparo Expte 40180. www.juscorrientes.gov.ar -consultado el 10/05/13-. 